\title{
A linearized kinetic theory of spin-1/2 particles in magnetized plasmas
}

\author{
J. Lundin and G. Brodin \\ Department of Physics, UmeåUniversity, SE-901 87 Umeå, Sweden
}

\begin{abstract}
We have considered linear kinetic theory including the electron spin properties in a magnetized plasma. The starting point is a mean field Vlasov-like equation, derived from a fully quantum mechanical treatment, where effects from the electron spin precession and the magnetic dipole force is taken into account. The general conductivity tensor is derived, including both the free current contribution, as well as the magnetization current associated with the spin contribution. We conclude the paper with an extensive discussion of the quantum-mechanical boundary where we list parameter conditions that must be satisfied for various quantum effects to be influential.
\end{abstract}

PACS numbers: $52.25 . \mathrm{Dg}, 52.25 . \mathrm{Xz}$

\section{INTRODUCTION}

Recently there has been much interest in the properties of quantum plasmas, see e.g. Refs. [1 12]. The research has been motivated by applications to e.g. quantum wells [12], plasmonics [9, 13], spintronics [14], astrophysics [15] and ultra-cold plasmas [16]. Quantum plasma effects has also been measured in solid density target experiments [17]. Within fluid theory, the theoretical models applied cover effects such as particle dispersion (the Bohm-de Broglie potential), and Fermi pressure. The magnetic dipole force and the magnetization current associated with the electron spin has also been captured within fluid models, with a macroscopic spin density as an extra dependent variable [7]. More accurate models applying kinetic theory has also been studied [6, 10, 18, 19]. In the absence of spin effects, a kinetic evolution equation can be derived for the Wigner function [1]. For long scale-lengths this equation reduces to the classical Vlasov equation.

Including the spin degrees of freedom, it was recently shown [6] that a Wigner transform in regular phase space and a Q-transform in spin space produces a physically attractive evolution equation for a scalar distribution function. If the magnetic moment of the particles is put to zero, the ordinary Wigner equation is immediately recovered. Here we will study the opposite limit, however. For wavelengths much longer than the characteristic de Broglie length of the particles, the quantum effects associated with particle dispersive effects (de-localized wavefunctions) disappear, and the evolution equation is much simplified. The remaining quantum effects are all due to the electron spin, explicitly due to terms being proportional to the magnetic moment in the dynamic equations, or indirectly due to the background distribution function obeying Fermi-Dirac statistic. The kinetic model obtained from the long-scale limit of a fully quantum mechanical treatment is a slight generalization of a semi-classical spin model presented in Ref. [10], where the spin vector complements the regular phase-space variables as an independent variable [6, 10]. In Ref. [10] it was also shown that new wave modes appear in a magnetized plasma due to the combined dynamics of the magnetic dipole force and the spin precession. Here we will extend the linear analysis made in that work in several directions:

1. By considering the case of general wave propagation (i.e. an arbitrary direction of propagation and a general wave polarization).

2. By not making any restrictions on the wave frequency domain.

3. By using a somewhat more accurate model, derived from a fully quantum mechanical treatment.

4. By presenting a more thorough discussion of the classical - quantum mechanical boundary.

The theory turns out to produce a Hermitian structure for the general conductivity tensor, including the free and magnetization currents, where all non-Hermitian contribution are associated with the poles. Contrary to common quantum effects associated with particle dispersion (de-localized wave functions), the effects due to the electron spin does not necessarily vanish in a plasma of moderate density and temperature. A low temperature and a high density tend to make spin effects more important (similarly to the quantum effects described in e.g. Refs. [1, 2] ), but a strong magnetic field or wave particle resonances can modify this picture considerably. As will be discussed in some detail in Section III, we point out that there is a large number of dimensionless parameters that together captures the relative significance of spin effects in different plasma regimes. Furthermore, in order to demonstrate the usefulness of the general conductivity tensor, in Section IV we present an analysis of the transition from wave damping to instability, when free energy is added to the the spin-degrees of freedom in the background distribution function. Specifically, it is shown that a background distribution function with equal population of the spin-up and spin-down states states leads to an instability. 


\section{THE CONDUCTIVITY TENSOR}

For long spatial scale-length the evolution of a (quasi) distribution function $f(\mathbf{r}, \mathbf{v}, \hat{\mathbf{s}}, t)$ for electrons is described by the Vlasov-like equation [6]

$$
\frac{\partial f}{\partial t}+\mathbf{v} \cdot \nabla_{\mathbf{x}} f+\left[\frac{q_{e}}{m_{e}}(\mathbf{E}+\mathbf{v} \times \mathbf{B})+\frac{\mu_{e}}{m_{e}} \nabla_{\mathbf{x}}\left(\hat{\mathbf{s}} \cdot \mathbf{B}+\mathbf{B} \cdot \nabla_{\hat{\mathbf{s}}}\right)\right] \cdot \nabla_{\mathbf{v}} f+\frac{2 \mu_{e}}{\hbar}(\hat{\mathbf{s}} \times \mathbf{B}) \cdot \nabla_{\hat{\mathbf{s}}} f=0,
$$

where $q_{e}=-e$ is the charge of an electron, $\mu_{e}=-(g / 2) e \hbar / 2 m_{e}$ is the electron magnetic moment (note that the sign is included) and $g=2.002319$ is the electron spin $g$ factor, $\hat{\mathbf{s}}$ is the unit spin vector, $\hbar$ is Planck's constant divided by $2 \pi$, and $m_{e}$ is the electron mass. The distribution function $f$ is normalized such that $\int f d^{2} s d^{3} v=n$ with $n$ being the number density. As for spin variables it is convenient to use ordinary spherical coordinates, i.e. $d^{2} s=\sin \theta_{s} d \theta_{s} d \varphi_{s}$.

In the derivation of Eq. (11) found in Ref. [6], trigonometric operators are expanded in powers of $\hbar$. Consequently, Eq. (11) is the long scale limit of a more complete quantum description, where long scale here means that length scales are larger than the characteristic de Broglie wavelength of the electrons. Upon disregarding spin effects, the opposite limit of the full quantum theory, i.e. for short length scales, is the well-known evolution equation for the Wigner function [1]. The spin term proportional to $\hat{\mathbf{s}} \cdot \mathbf{B}$ in Eq. (1) is due to the magnetic dipole force, and the term proportional to $\hat{\mathbf{s}} \times \mathbf{B}$ owes to the spin precession. Both these terms are also found in the semi-classical version of Eq. (11), see Ref. [10]. The spin term proportional to $\mathbf{B} \cdot \nabla_{\hat{\mathbf{s}}}$, however, is a fully quantum mechanical effect. Essentially this term can be viewed as a modification of the magnetic dipole force that occurs due to the spread out nature of the spin probability distribution.

By solving Eq. (1) we may find an expression for the distribution function $f$ from which we can construct the free current, $\mathbf{J}_{f}$, and the magnetization current density, $\mathbf{J}_{M}$, according to [6]

$$
\begin{aligned}
\mathbf{J} & =\mathbf{J}_{f}+\mathbf{J}_{M} \\
& =\mathbf{J}_{f}+\nabla_{\mathbf{x}} \times \mathbf{M} \\
& =q_{e} \int \mathbf{v} f d^{2} s d^{3} v+\nabla_{\mathbf{x}} \times\left(\mu_{e} \int 3 \hat{\mathbf{s}} f d^{2} s d^{3} v\right) .
\end{aligned}
$$

In this section we only consider the electron contribution to the current density. The ion contribution may typically be treated classically (because ion spin effects are suppressed compared to electron spin effects due the heavy ion mass), and its contribution may be found in standard literature, see e.g. Ref. [20]. The derivation given below may, however, easily be generalized to also apply for arbitrary spin-1/2 particles through the appropriate substitution of charge, mass and magnetic moment.

Equations (11) and (2) gives, together with the Maxwell's equations, a closed system of equations that can be solved to describe the dynamics of a plasma where quantum effects of the electron spin are captured. Below we will derive the conductivity tensor $\sigma_{i j}$, defined as $J_{i}=\sigma_{i j} E_{j}$, for such a system [21]. With the conductivity tensor known, it is straightforward to construct a dispersion matrix and find the dispersion relations for arbitrary wave modes. Next, we linearize the kinetic Eq. (11) according to $f=f_{0}+f_{1}$ and $\mathbf{B}=\mathbf{B}_{0}+\mathbf{B}_{1}$, where the subscript 0 denotes an unperturbed quantity and the subscript 1 denotes a perturbation, and we take $\mathbf{B}_{0}=B_{0} \hat{\mathbf{z}}$. Before proceeding, let us point out a few quantum effects that may be contained already in the unperturbed distribution function:

1. Fermi-Dirac statistics: This effect is well-known. Here we just note that for a plasma of moderate density and temperature this effect may be suppressed, as a large (negative) chemical potential (that applies for $\left.\hbar^{2} n_{0}^{2 / 3} / m_{e} k_{B} T \ll 1\right)$ turns the thermodynamic equilibrium distribution into the classical Maxwellian.

2. Landau-quantization: The quantization of perpendicular energy states becomes important in the regime of very strong magnetic fields, or very low temperatures, when $\hbar\left|\omega_{c e}\right| / k_{B} T \rightarrow 1$, where $k_{B}$ is Boltzmann's constant and $\omega_{c e}=-e B_{0} / m_{e}$ is the electron cyclotron frequency.

3. Spin-splitting: The two spin states, up- and down relative to the magnetic field, clearly have different probability distributions in spin space. As a result, the general time-independent distribution function can be written as $f_{0}=f_{0+}+f_{0-}$ with $f_{0 \pm}=(1 / 4 \pi) F_{0 \pm}(\mathbf{v})\left(1 \pm \cos \theta_{s}\right)$, where for a time-independent distribution function $F_{0 \pm}$ can be arbitrary functions of $\left(v_{\perp}, v_{z}\right)$, and $F_{0 \pm}$ is normalized such that $\int F_{0 \pm} d^{3} v=n_{0 \pm}$ with $n_{0 \pm}$ being the number densities of the spin up/down states respectively. The positive spin state here means that the spin points in the direction parallel to the magnetic field, which means that the magnetic moment points in the opposite direction. Note that with this definition, the lower energy state is the spin state with negative index, i.e. $n_{0-}>n_{0+}$ in case the background distribution $f_{0}$ describes a thermodynamic equilibrium. 
For a full quantum mechanical expression of the thermodynamic equilibrium distribution, see Ref. [6]. However, in the present manuscript we will focus on the effects due to the dynamical equations, rather than the initial conditions briefly discussed above. Still, we note that since the spin-distribution has no classical limit point 3 above cannot be ignored. Since the present collision-free model has no mechanism for spin-flips to occur, we also note that the number of particles with spin-up $N_{+}=\int f_{0+} d^{2} s d^{3} v d^{3} r$ and the number of particles with spin down $N_{-}=\int f_{0-} d^{2} s d^{3} v d^{3} r$ are conserved quantities. Furthermore, in thermodynamic equilibrium the spin-up and spin-down number densities $n_{0 \pm}=\int f_{0 \pm} d^{2} s d^{3} v$ are related by $\left(n_{0+}-n_{0-}\right) /\left(n_{0+}+n_{0-}\right)=\tanh \left(\mu_{e} B_{0} / k_{B} T\right)$. Since the evolution equation is the same, independent of the unperturbed spin state, it is convenient to wait with the split of $f_{0}$ into $f_{0 \pm}$ to the final stages of the calculation.

After linearization, Eq. (1) is written as

$$
\begin{aligned}
& \frac{\partial f_{1}}{\partial t}+\mathbf{v} \cdot \nabla_{\mathbf{x}} f_{1}+\frac{q_{e}}{m_{e}}\left(\mathbf{v} \times \mathbf{B}_{0}\right) \cdot \nabla_{\mathbf{v}} f_{1}+\frac{2 \mu_{e}}{\hbar}\left(\hat{\mathbf{s}} \times \mathbf{B}_{0}\right) \cdot \nabla_{\hat{\mathbf{s}}} f_{1} \\
= & -\left[\frac{q_{e}}{m_{e}}\left(\mathbf{E}+\mathbf{v} \times \mathbf{B}_{1}\right)+\frac{\mu_{e}}{m_{e}} \nabla_{\mathbf{x}}\left(\hat{\mathbf{s}} \cdot \mathbf{B}_{1}+\mathbf{B}_{1} \cdot \nabla_{\hat{\mathbf{s}}}\right)\right] \cdot \nabla_{\mathbf{v}} f_{0}-\frac{2 \mu_{e}}{\hbar}\left(\hat{\mathbf{s}} \times \mathbf{B}_{1}\right) \cdot \nabla_{\hat{\mathbf{s}}} f_{0} .
\end{aligned}
$$

Next we make a plane wave ansatz of the perturbed parameters according to $f_{1}=\tilde{f}_{1} \exp [i(\mathbf{k} \cdot \mathbf{x}-\omega t)]$, etc. Without loss of generality, we define the wavevector as $\mathbf{k}=k_{\perp} \hat{\mathbf{x}}+k_{z} \hat{\mathbf{z}}$. We also choose to express the velocity in cylindrical coordinates $\left(v_{\perp}, \varphi_{v}, v_{z}\right)$ such that $d^{3} v=v_{\perp} d v_{\perp} d \varphi_{v} d v_{z}$, and expand $f_{1}$ in eigenfunctions to the operator of the right hand side

$$
\tilde{f}_{1}=\sum_{a=-\infty}^{\infty} \sum_{b=-\infty}^{\infty} g_{a b}\left(v_{\perp}, v_{z}, \theta_{s}\right) \psi_{a}\left(\varphi_{v}, v_{\perp}\right) \frac{1}{\sqrt{2 \pi}} \exp \left(-i b \varphi_{s}\right)
$$

where

$$
\begin{aligned}
\psi_{a}\left(\varphi_{v}, v_{\perp}\right) & =\frac{1}{\sqrt{2 \pi}} \exp \left[-i\left(a \varphi_{v}-k_{\perp} v_{\perp} \sin \varphi_{v} / \omega_{c e}\right)\right] \\
& =\frac{1}{\sqrt{2 \pi}} \sum_{l=-\infty}^{\infty} \mathcal{J}_{l}\left(\frac{k_{\perp} v_{\perp}}{\omega_{c e}}\right) \exp \left[i(l-a) \varphi_{v}\right]
\end{aligned}
$$

where $\mathcal{J}_{l}(x)$ is a Bessel function of the first kind. We may then note the following simplifying relations;

$$
\begin{aligned}
\frac{q_{e}}{m_{e}}\left(\mathbf{v} \times \mathbf{B}_{0}\right) \cdot \nabla_{\mathbf{v}} f_{1} & =-\omega_{c e} \frac{\partial f_{1}}{\partial \varphi_{v}} \\
\frac{2 \mu_{e}}{\hbar}\left(\hat{\mathbf{s}} \times \mathbf{B}_{0}\right) \cdot \nabla_{\hat{\mathbf{s}}} f_{1} & =-\omega_{c g} \frac{\partial f_{1}}{\partial \varphi_{s}}
\end{aligned}
$$

where $\omega_{c g}=2 \mu_{e} B_{0} / \hbar$ is the spin precession frequency. Moreover, for simplicity we assume an isotropic distribution function on the form $f_{0}=f_{0}\left(v^{2}, \theta_{s}\right)$. For this case we may drop the term $\left(\mathbf{v} \times \mathbf{B}_{1}\right) \cdot \nabla_{\mathbf{v}} f_{0}$. We may also take advantage of the relations $\left(\partial f_{0} / \partial v_{\perp}\right) / 2 v_{\perp}=\left(\partial f_{0} / \partial v_{z}\right) / 2 v_{z}=\partial f_{0} / \partial v^{2}$.

Using the eigenfunction expansion of $\tilde{f}_{1}$ (Eq. (4)) in the linearized Vlasov equation (3), multiplying the resulting equation with $\psi_{a}^{*} e^{i b \varphi_{s}} / \sqrt{2 \pi}$ (where the star denotes complex conjugate) and integrating over $\varphi_{v}$ and $\varphi_{s}$, we find the equation

$$
i\left(\omega-k_{z} v_{z}-a \omega_{c e}-b \omega_{c g}\right) g_{a b}=I_{a b}\left(v_{\perp}, v_{z}, \varphi_{s}\right)
$$

where

$$
I_{a b}=\int_{0}^{2 \pi} \int_{0}^{2 \pi} d \varphi_{v} d \varphi_{s}\left[\left(\frac{q_{e}}{m_{e}} \tilde{\mathbf{E}}+\frac{\mu_{e}}{m_{e}} \nabla_{\mathbf{x}}\left(\hat{\mathbf{s}} \cdot \tilde{\mathbf{B}}_{1}+\tilde{\mathbf{B}}_{1} \cdot \nabla_{\hat{\mathbf{s}}}\right)\right) \cdot \nabla_{\mathbf{v}} f_{0}+\frac{2 \mu_{e}}{\hbar}\left(\hat{\mathbf{s}} \times \tilde{\mathbf{B}}_{1}\right) \cdot \nabla_{\hat{\mathbf{s}}} f_{0}\right] \psi_{a}^{*} \frac{1}{\sqrt{2 \pi}} \exp \left(i b \varphi_{s}\right) .
$$

From Eq. (7a) we can then solve $g_{a b}$ and thereby construct $\tilde{f}_{1}$ in terms of $f_{0}$. It may be useful to note that the vector 
products in (7b) are;

$$
\begin{aligned}
\tilde{\mathbf{E}} \cdot \nabla_{\mathbf{v}} f_{0} & =2\left(E_{x} v_{\perp} \cos \varphi_{v}+E_{y} v_{\perp} \sin \varphi_{v}+E_{z} v_{z}\right) \frac{\partial f_{0}}{\partial v^{2}}, \\
\nabla_{\mathbf{x}}\left(\hat{\mathbf{s}} \cdot \tilde{\mathbf{B}}_{1}\right) \cdot \nabla_{\mathbf{v}} f_{0} & =2 i\left(k_{z} v_{z}+k_{\perp} v_{\perp} \cos \varphi_{v}\right)\left(B_{x} \sin \theta_{s} \cos \varphi_{s}+B_{y} \sin \theta_{s} \sin \varphi_{s}+B_{z} \cos \theta_{s}\right) \frac{\partial f_{0}}{\partial v^{2}}, \\
\nabla_{\mathbf{x}}\left(\tilde{\mathbf{B}}_{1} \cdot \nabla_{\hat{\mathbf{s}}}\right) \cdot \nabla_{\mathbf{v}} f_{0} & =2 i\left(k_{z} v_{z}+k_{\perp} v_{\perp} \cos \varphi_{v}\right)\left(B_{x} \cos \theta_{s} \cos \varphi_{s}+B_{y} \cos \theta_{s} \sin \varphi_{s}-B_{z} \sin \theta_{s}\right) \frac{\partial^{2} f_{0}}{\partial \theta_{s} \partial v^{2}}, \\
\left(\hat{\mathbf{s}} \times \tilde{\mathbf{B}}_{1}\right) \cdot \nabla_{\hat{\mathbf{s}}} f_{0} & =\left(B_{x} \sin \varphi_{s}-B_{y} \cos \varphi_{s}\right) \frac{\partial f_{0}}{\partial \theta_{s}} .
\end{aligned}
$$

With the relations (5) and (8a)-(8d), the integration in (7b) can be performed in a tedious but straight forward manner (some useful integrals are found in appendix A. Eqs. (A1a)-(A1c), and through Eq. (4) the resulting expression for $\tilde{f}_{1}$ becomes

$$
\tilde{f}_{1}=\sum_{a=-\infty}^{\infty} \sum_{l=-\infty}^{\infty} \mathcal{J}_{l} e^{i(l-a) \varphi_{v}}\left(\frac{A e^{i \varphi_{s}}}{\omega-k_{z} v_{z}-a \omega_{c e}+\omega_{c g}}+\frac{B}{\omega-k_{z} v_{z}-a \omega_{c e}}+\frac{C e^{-i \varphi_{s}}}{\omega-k_{z} v_{z}-a \omega_{c e}-\omega_{c g}}\right)
$$

with

$$
\begin{aligned}
A & =\frac{\mu_{e}}{m_{e} \omega}\left(-i k_{z} E_{x}-k_{z} E_{y}+i k_{\perp} E_{z}\right)\left[-\frac{m_{e}}{\hbar} \frac{\partial f_{0}}{\partial \theta_{s}}+\left(a \omega_{c e}+k_{z} v_{z}\right)\left(\sin \theta_{s} \frac{\partial f_{0}}{\partial v^{2}}+\cos \theta_{s} \frac{\partial^{2} f_{0}}{\partial \theta_{s} \partial v^{2}}\right)\right] \mathcal{J}_{a} \\
B & =2 \frac{\mu_{e}}{m_{e} \omega} E_{y} k_{\perp}\left(a \omega_{c e}+k_{z} v_{z}\right)\left(\cos \theta_{s} \frac{\partial f_{0}}{\partial v^{2}}-\sin \theta_{s} \frac{\partial^{2} f_{0}}{\partial \theta_{s} \partial v^{2}}\right) \mathcal{J}_{a}-2 i \frac{q_{e}}{m_{e}}\left(E_{x} a \frac{\omega_{c e}}{k_{\perp}} \mathcal{J}_{a}+i E_{y} v_{\perp} \mathcal{J}_{a}^{\prime}+E_{z} v_{z} \mathcal{J}_{a}\right) \frac{\partial f_{0}}{\partial v^{2}} \\
C & =\frac{\mu_{e}}{m_{e} \omega}\left(i k_{z} E_{x}-k_{z} E_{y}-i k_{\perp} E_{z}\right)\left[\frac{m_{e}}{\hbar} \frac{\partial f_{0}}{\partial \theta_{s}}+\left(a \omega_{c e}+k_{z} v_{z}\right)\left(\sin \theta_{s} \frac{\partial f_{0}}{\partial v^{2}}+\cos \theta_{s} \frac{\partial^{2} f_{0}}{\partial \theta_{s} \partial v^{2}}\right)\right] \mathcal{J}_{a} .
\end{aligned}
$$

Here, the argument of the Bessel functions is $k_{\perp} v_{\perp} / \omega_{c e}$. In obtaining Eq. (9) we have used $\nabla \times \mathbf{E}=-\partial \mathbf{B} / \partial t$ to relate the magnetic field to the electric field, as well as the Bessel function identities $\mathcal{J}_{a+1}(x)+\mathcal{J}_{a-1}(x)=2 a \mathcal{J}_{a}(x) / x$ and $\mathcal{J}_{a-1}(x)-\mathcal{J}_{a+1}(x)=2 \mathcal{J}_{a}^{\prime}(x)$.

With $\tilde{f}_{1}$ known in terms of $f_{0}$, we proceed by splitting $f_{0}$ into its spin states, $f_{0}=f_{0+}+f_{0}$, and construct the free current

$$
\begin{aligned}
\mathbf{J}_{f} & =\sum_{\nu=+,-} q_{e} \int \mathbf{v} f_{1 \nu} d^{2} s d^{3} v \\
& =\sum_{\nu=+,-} q_{e} \int \sum_{a=-\infty}^{\infty}\left[a \frac{\omega_{c e}}{k_{\perp}} \mathcal{J}_{a} \hat{\mathbf{x}}-i v_{\perp} \mathcal{J}_{a}^{\prime} \hat{\mathbf{y}}+v_{z} \mathcal{J}_{a} \hat{\mathbf{z}}\right] \tilde{B}_{\nu} d^{2} s d^{3} v
\end{aligned}
$$

as well as the magnetization current

$$
\begin{aligned}
\mathbf{J}_{M} & =\sum_{\nu=+,-} \nabla \times\left(3 \mu_{e} \int \mathbf{s} f_{1 \nu} d^{2} s d^{3} v\right) \\
& =\sum_{\nu=+,-} \frac{3}{2} \mu_{e} \int \sum_{a=-\infty}^{\infty} \mathcal{J}_{a} \sin \theta_{s}\left[\left(\tilde{A}_{\nu}-\tilde{C}_{\nu}\right) k_{z} \hat{\mathbf{x}}+i\left(\left(\tilde{A}_{\nu}+\tilde{C}_{\nu}\right) k_{z}-\frac{2 \cos \theta_{s}}{\sin \theta_{s}} \tilde{B}_{\nu} k_{\perp}\right) \hat{\mathbf{y}}-\left(\tilde{A}_{\nu}-\tilde{C}_{\nu}\right) k_{\perp} \hat{\mathbf{z}}\right] d^{2} s d^{3} v
\end{aligned}
$$

where we have performed the $\varphi_{v}$ and $\varphi_{s}$ integration and immediately reintroduced the full integration element for convenience of a compact notation. We have also included the denominators in (9) in $\tilde{A}_{\nu}$ by defining $\tilde{A}_{\nu}=A_{\nu} /(\omega-$ $\left.k_{z} v_{z}-a \omega_{c e}+\omega_{c g}\right)$, and similarly for $\tilde{B}_{\nu}$ and $C_{\nu}$.

Before we construct the conductivity tensor, we note that we can simplify things yet further by writing $f_{0 \pm}=$ $(1 / 4 \pi)\left(1 \pm \cos \theta_{s}\right) F_{0 \pm}\left(v^{2}\right)$. We may then perform the $\theta_{s}$ integrations in Eqs. (10) and (11) (a useful integral is found in Appendix A] Eq. (A2)), and construct the conductivity tensor which may be written as

$$
\sigma_{i j}=\sum_{\nu=+,-} \sum_{a=-\infty}^{\infty} \int\left[\frac{X_{(\nu) i j}^{(\mathrm{sp})}}{\omega-k_{z} v_{z}-a \omega_{c e}+\omega_{c g}}+\frac{Y_{(\nu) i j}^{(\mathrm{cl})}+Y_{(\nu) i j}^{(\mathrm{sp})}}{\omega-k_{z} v_{z}-a \omega_{c e}}+\frac{Z_{(\nu) i j}^{(\mathrm{sp})}}{\omega-k_{z} v_{z}-a \omega_{c e}-\omega_{c g}}\right] d^{3} v
$$


where

$$
Y_{(\nu) i j}^{(\mathrm{cl})}=2 \frac{q_{e}^{2}}{m_{e}} \frac{\partial F_{0 \nu}}{\partial v^{2}} \times\left(\begin{array}{ccc}
-i a^{2} \frac{\omega_{c e}^{2}}{k_{\perp}^{2}} \mathcal{J}_{a}^{2} & a \frac{\omega_{c e}}{k_{\perp}} v_{\perp} \mathcal{J}_{a} \mathcal{J}_{a}^{\prime} & -i a \frac{\omega_{c e}}{k_{\perp}} v_{z} \mathcal{J}_{a}^{2} \\
-a \frac{\omega_{c e}}{k_{\perp}} v_{\perp} \mathcal{J}_{a} \mathcal{J}_{a}^{\prime} & -i v_{\perp}^{2} \mathcal{J}_{a}^{\prime 2} & -v_{\perp} v_{z} \mathcal{J}_{a} \mathcal{J}_{a}^{\prime} \\
-i a \frac{\omega_{c e}}{k_{\perp}} v_{z} \mathcal{J}_{a}^{2} & v_{\perp} v_{z} \mathcal{J}_{a} \mathcal{J}_{a}^{\prime} & -i v_{z}^{2} \mathcal{J}_{a}^{2}
\end{array}\right)
$$

is the classical contribution, and the spin contributions are

$$
Y_{(\nu) i j}^{(\mathrm{sp})}=-2 \mu_{e} \frac{q_{e}}{m_{e}} \frac{\partial F_{0 \nu}}{\partial v^{2}} \times\left(\begin{array}{ccc}
0 & -\nu a \omega_{c e} \mathcal{J}_{a}^{2} & 0 \\
\nu a \omega_{c e} \mathcal{J}_{a}^{2} & i \frac{\mu_{e}}{q_{e}} \frac{k_{\perp}^{2}}{\omega}\left(a \omega_{c e}+k_{z} v_{z}\right) \mathcal{J}_{a}^{2} & \nu k_{\perp} v_{z} \mathcal{J}_{a}^{2} \\
& +\nu i\left(\omega+a \omega_{c e}+k_{z} v_{z}\right) \frac{k_{\perp} v_{\perp}}{\omega} \mathcal{J}_{a} \mathcal{J}_{a}^{\prime} & \\
0 & -\nu k_{\perp} v_{z} \mathcal{J}_{a}^{2} & 0
\end{array}\right)
$$

together with

$$
\begin{aligned}
& X_{(\nu) i j}^{(\mathrm{sp})}=i \frac{\mu_{e}^{2}}{\hbar \omega}\left(\nu F_{0 \nu}+\frac{\hbar}{m_{e}}\left(a \omega_{c e}+k_{z} v_{z}\right) \frac{\partial F_{0 \nu}}{\partial v^{2}}\right) \mathcal{J}_{a}^{2} M_{i j} \\
& Z_{(\nu) i j}^{(\mathrm{sp})}=i \frac{\mu_{e}^{2}}{\hbar \omega}\left(-\nu F_{0 \nu}+\frac{\hbar}{m_{e}}\left(a \omega_{c e}+k_{z} v_{z}\right) \frac{\partial F_{0 \nu}}{\partial v^{2}}\right) \mathcal{J}_{a}^{2} M_{i j}^{*}
\end{aligned}
$$

where

$$
M_{i j}=\left(\begin{array}{ccc}
-k_{z}^{2} & i k_{z}^{2} & k_{\perp} k_{z} \\
-i k_{z}^{2} & -k_{z}^{2} & i k_{\perp} k_{z} \\
k_{\perp} k_{z} & -i k_{\perp} k_{z} & -k_{\perp}^{2}
\end{array}\right)
$$

Given the conductivity tensor (12), the general dispersion relation is obtained in the same way as in the classical case, i.e. $\operatorname{det} D_{i j}=0$, with $D_{i j}=\delta_{i j}-k_{i} k_{j} c^{2} / \omega^{2}-i \sigma_{i j} / \varepsilon_{0} \omega$. Analogously to the classical case the conductivity tensor has a Hermitian structure $\left(\sigma_{i j}=-\sigma_{j i}^{*}\right)$ if the pole-contributions (associated with the denominators $\left(\omega-k_{z} v_{z}-a \omega_{c e}-b \omega_{c g}\right)$ ) are dropped. It should be noted the Hermitian structure of the conductivity tensor does not follow trivially from Eqs. (10) and (11). A detailed discussion on this is found in appendix B.

In appendix C, we derive the short Larmor radius limit of the conductivity tensor (12), i.e. the limit in which the argument of the Bessel functions are small, $k_{\perp} v_{\perp} / \omega_{c e} \ll 1$.

\section{THE CLASSICAL - QUANTUM MECHANICAL BOUNDARY}

In addition to the spin phenomena studied here, using Eq. (1), kinetic quantum plasma phenomena associated with non-localized wave functions can be studied using the Wigner function, see e.g. Ref. [1]. Those quantum effects prove to be important for wavelengths shorter than the thermal de Broglie wavelength. However, collective effects are typically negligible for such short wavelengths, unless the parameter $d_{1}=\hbar \omega_{p} / k_{B} T_{\mathrm{m}}$ approaches unity, where $\omega_{p}=\left(n_{0} e^{2} / \varepsilon_{0} m_{e}\right)^{1 / 2}$ is the plasma frequency. Here $T_{\mathrm{m}}$ is the largest quantity of the Fermi temperature $T_{\mathrm{F}}$ and the thermodynamic temperature $T$. Thus it is safe to say that the quantum effects that are not associated with the spin requires either a low temperature and/or a high density. In addition to quantum effects associated with de-localized wavefunctions, there are those associated with the Fermi pressure. Naturally the relative importance of this effect is captured by the parameter $d_{2}=T_{\mathrm{F}} / T=\left(3 \pi^{2}\right)^{2 / 3} \hbar^{2} n^{2 / 3} / 2 m_{e} k_{B} T$, which also requires a low temperature and/or a high density plasma, although the scaling differs somewhat from that of $d_{1}$. In linear theory, given a classical kinetic dispersion relation for a thermodynamic equilibrium system, the effects of the Fermi pressure is relatively trivial, since it can be deduced simply by replacing an unperturbed Maxwellian distribution with the Fermi-Dirac distribution. Estimating when the electron spin effects is of importance is somewhat more complicated, as the relevant dimensionless parameter now also involves the magnetic field strength and the wave-number and wave-frequency. As will be verified below, from the expression for the conductivity tensor components (12), we point out a set of important 
parameters determining the relative strength of spin effects. Throughout this section we will assume the presence of ions described by a classical contribution to the conductivity tensor, see e.g. Ref. [20] and electrons described by Eq. (12).

- The Zeeman energy over thermal energy, $d_{3}=\mu_{e} B_{0} / k_{B} T=\hbar \omega_{c e} / k_{B} T$. This parameters enters for several reasons. The simplest one is that it determines the ratio between the spin-up and -down populations (i.e. $F_{0+}$ versus $F_{0-}$ in (12) $)$ in thermodynamic equilibrium.

- $d_{4}=\hbar k^{2} / m_{e} \omega$. This parameter is important since it determines the relative strength of the magnetic dipole force as compared to the force from the electric field. For systems that allow short-wavelength low-frequency modes this is of particular significance.

- $d_{5}=\hbar \omega_{c e} / m_{i} c_{A}^{2}$, where $c_{A}=\left(B_{0}^{2} / \mu_{0} m_{i} n_{0}\right)^{1 / 2}$ is the Alfvén velocity. This parameter can appear for several reasons. A relatively simple example is that it gives the relative strength of the magnetic dipole force to the Lorentz force, evaluated for ion-cyclotron waves.

- $d_{6}=\hbar \omega_{p} / m_{e} c^{2}$. This parameter describes the relative magnetic permeability due to the spin, for a given spin state. When it approaches unity the density is so high that a relativistic treatment is called for. Thus the regime $d_{6}>1$ is strictly not allowed by our treatment. However, as we will see below, the parameter $d_{6}$ still have a certain significance, also for our non-relativistic case.

Although the parameters $d_{1}$ and $d_{2}$ has not been directly associated with the electron spin, it should be noted that these two quantities can also be involved when determining the relative importance of spin for a specific wavemode. In some cases it is a combination of the basic parameters $d_{1}-d_{6}$ that gives the ratio of the spin and classical contribution. In order to illustrate the situation further, we will briefly discuss some specific examples.

Magnetosonic waves: For usual ideal MHD waves there are the shear Alfvén wave with $E_{x} \neq 0$, and the fast and slow magnetosonic waves with $E_{y, z} \neq 0$. The latter wave mode is determined by the components $\sigma_{y z}\left(=-\sigma_{z y}^{*}\right)$, $\sigma_{y y}$ and $\sigma_{z z}$. Assuming that the plasma is strongly magnetized or has a low temperature, we consider the case when $d_{3}$ approaches unity, but all other quantum parameters are small. Considering the MHD-regime it is then found that the ratio of the spin contribution to $\sigma_{y z}\left(\right.$ from $\left.\left(Y_{i j}^{(\mathrm{sp})}\right)\right)$ to the classical contribution (from $\left.\left(Y_{i j}^{(\mathrm{cl})}\right)\right)$ is $\sigma_{y z}^{(\mathrm{sp})} / \sigma_{y z}^{(\mathrm{cl})} \sim d_{3}^{2}$. For a low-beta plasma this mainly affects the slow magnetosonic mode, but for a high beta plasma both the fast and slow magnetosonic mode is much affected in this regime. This example mainly applies to strongly magnetized plasmas that occur in astrophysics, e.g. pulsars and magnetars. If experiments on ultra-cold plasmas [16] were extended to include strongly magnetized plasmas, this could also be a regime of relevance.

Ion-cyclotron waves: When ordinary MHD waves (e.g. shear Alfvén waves and compressional Alfvén waves) have their wavelengths shortened to $k_{z} \sim \omega_{c i} / c_{A}$ and $k_{\perp} \sim \omega_{c i} / c_{A}$ where $\omega_{c i}$ is the ion-cyclotron frequency, the frequencies are increased up to $\omega \sim \omega_{c i}$, and the waves become dispersive. For arbitrary directions of propagation, all components of the conductivity tensor are of relevance, although the classical expression for $\sigma_{x z}$ (and $\sigma_{z x}$ ) is usually possible to put to zero. For definiteness we will make a comparison of $\sigma_{x x}$, but other components would work as well. We consider a regime of relatively high density, but with a magnetic field that is not particularly strong. For example, $n_{0}=10^{21} \mathrm{~cm}^{-3}$ and $B_{0}=10^{-4} \mathrm{~T}$. We note that this regime means that $c_{A}^{2} \ll c^{2}$ and for a moderate temperature (such that the Bessel functions can be Taylor expanded to lowest order) the only large quantum parameter is $d_{5}$ (and possibly $d_{4}$ ). Evaluating the classical part of $\sigma_{x x}$ (that comes from the ions) and the spin part of the same component (that comes from the terms proportional to $X_{i j}^{(\mathrm{sp})}$ (or $Z_{i j}^{(\mathrm{sp})}$ ) with $a= \pm 1$ such that the relevant denominators in (12) becomes $\omega-k_{z} v_{z} \pm\left(\omega_{c e}-\omega_{c g}\right)$, we find the ratio $\sigma_{x x}^{(\mathrm{sp})} / \sigma_{x x}^{(\mathrm{cl})}=d_{5}^{2}$, where we have used that $\left|\omega_{c e}-\omega_{c g}\right|$ is of the order of the ion-cyclotron frequency $\omega_{c i}$. In general all of the different ion-cyclotron modes are much affected when $d_{5} \rightarrow 1$ [22].

Whistler waves: Next we focus on the case of whistler waves, $\omega \lesssim\left|\omega_{c e}\right|$, where the electron conductivity is dominant also for the classical contribution. Furthermore we let $k_{\perp} v_{\mathrm{th}} /\left|\omega_{c e}\right| \ll 1$, such that the Bessel functions can be expanded, and we note that we are considering the regime of short perpendicular wavelengths, $k_{\perp}<\left|\omega_{p e}\right| / c$. The most relevant components here are $\sigma_{x x}, \sigma_{y y}$ as well as $\sigma_{x y}$. The dominant spin contribution comes from the $\sigma_{x y}$ component of $Y_{i j}^{(\mathrm{sp})}$, which should be compared to the $\sigma_{x y}$ component of $Y_{i j}^{(\mathrm{cl})}$. Using the thermodynamic equilibrium relation $\left(n_{0+}-n_{0-}\right) /\left(n_{0+}+n_{0-}\right)=\tanh \left(\mu_{e} B_{0} / k_{B} T\right) \approx \mu_{e} B_{0} / k_{B} T$, we then find the ratio $\sigma_{x y}^{(\mathrm{sp})} / \sigma_{x y}^{(\mathrm{cl})}=d_{3} d_{5}$, and similar comparisons can be made for other conductivity components in the whistler regime. Note that for a nonrelativistic Alfvén velocity we can simplify $d_{3} d_{5}=d_{6}\left(\hbar \omega_{p} / k_{B} T\right)$. Since it is the thermodynamic temperature that enters here (which determines the relative population of the two spin states), rather than the Fermi temperature, we can very well be in the non-relativistic regime $d_{6} \ll 1$ simultaneously as $d_{6}\left(\hbar \omega_{p} / k_{B} T\right) \sim 1$. For example, a plasma with density $n_{0}=10^{30} \mathrm{~cm}^{3}$ and $T=10^{5} \mathrm{~K}$ is nonrelativistic to a good approximation (i.e. $d_{6} \ll 1$ ) at the same time as $d_{3} d_{5} \sim 1$. 
The above brief examples illustrates some possibilities to get conductivity components due to the spin that are comparable to the classical ones in magnitude. Note that it is hard to give simple general guidelines when spin effects are important. For example, for a given temperature we may need a sufficiently strong magnetic field for spin to be important. We saw this in the example with magnetosonic waves, where the parameter $d_{3}$ should be of order unity. On the other hand, for a given density, and for certain wave modes, we may need a sufficiently weak magnetic field for spin to be important, as we saw in the example with ion-cyclotron waves involving the parameter $d_{5}$.

Furthermore, sometimes the spin terms are small compared to the classical terms, but still give a significant contribution. The reason is that a number of small but qualitatively new effects from the spin can be added to the dispersion relation. Thus, in order to get a somewhat deeper understanding of the classical - quantum mechanical transition, than mere estimates of magnitude can give, we will present a specific calculation in more detail. In the example below, we follow the assumptions made in Ref. [11]. We then focus on the regime $k_{\perp} v_{t h} /\left|\omega_{c e}\right| \ll 1$, such that the Bessel functions can be expanded and consider $d_{3} \ll 1$. As pointed out previously, the latter strong inequality condition holds for most plasmas, except for some strongly magnetized astrophysical objects [15]. Furthermore, to be specific, we consider waves with a polarization $\mathbf{E}=E_{y} \widehat{\mathbf{y}}$ and $\mathbf{B}=B_{x} \widehat{\mathbf{x}}+B_{z} \widehat{\mathbf{z}}=\left(k_{z} E_{y} / \omega\right) \widehat{\mathbf{x}}-\left(k_{\perp} E_{y} / \omega\right) \widehat{\mathbf{z}}$. For this polarization to be possible we must have $\sigma_{x y}, \sigma_{x z} \ll \sigma_{y y}$, which can be verified a posteriori. We keep the standard classical terms (see e.g. Ref. [20]) up to order $\omega_{c e}^{-2}$ in an $1 / \omega_{c e}$-expansion (for both ions and electrons), where the dimensionless expansion parameters are considered to be $\omega / \omega_{c e}, k_{z} v_{t h} / \omega_{c e}$ and $k_{\perp} v_{t h} / \omega_{c e}$. The spin effects are assumed to be smaller, due to $\hbar\left|\omega_{c e}\right| / k_{B} T \ll 1$, and we also consider the case $\omega /\left|\omega_{c e}-\omega_{c g}\right|_{c} \ll 1$. Accordingly we only include spin terms to zero:th order in the $1 / \omega_{c e}$-expansion. With these prerequisites the only spin effect that survives is the $z$-component of the magnetic dipole force, and its corresponding modification of the $y$-component of the magnetization current. For the given polarization the dispersion relation then reads

$$
\omega^{2}-k^{2} c^{2}-\frac{i \omega}{\varepsilon_{0}} \sigma_{y y}=0
$$

where $\sigma_{y y}$ is given by

$$
\sigma_{y y}=i \varepsilon_{0} \omega \frac{\omega_{p i}^{2}}{\omega_{c i}^{2}}+i \varepsilon_{0} \frac{\hbar^{2} k_{z}^{2} k_{\perp}^{2} \omega_{p e}^{2}}{4 \omega m_{e}^{2}} \int \frac{\widehat{F}_{0}}{\left(\omega-k_{z} v_{z}\right)^{2}} d v_{z}
$$

where all integrations except that over $d v_{z}$ has been carried out, and the normalization of the rescaled distribution function $\widehat{F}_{0}$ is given by $\int \widehat{F}_{0} d v_{z}=1$. In Eq. (14) the first term of the right hand side is the classical contribution from the ion free current, whereas the second term is due to the electron magnetization current. The dispersion relation (13) now immediately reduces to

$$
\omega^{2}=\frac{k^{2} c^{2}}{1+\omega_{p i}^{2} / \omega_{c i}^{2}}\left[1-\sin ^{2} \alpha \cos ^{2} \alpha \frac{\hbar^{2} k^{2} \omega_{p e}^{2}}{4 m_{e}^{2} c^{2}} \int \frac{\widehat{F}_{0}}{\left(\omega-k_{z} v_{z}\right)^{2}} d v_{z}\right]
$$

where $\sin ^{2} \alpha=k_{\perp}^{2} / k^{2}$. Firstly, we note that for ordinary densities in space and laboratory plasmas, the influence of the real value of $\omega$ is small and thus we can omit the spin term as well as the first term of Eq. (13) and write the standard dispersion relation for compressional Alfvén waves $\omega^{2}=k^{2} c_{A}^{2}$, where we have assumed $c_{A} \ll c$ (or $\left.\omega_{p i}^{2} / \omega_{c i}^{2} \gg 1\right)$. Furthermore, for $\operatorname{Re}(\omega)$ to be significantly changed by the spin effects, we need high densities and/or low temperatures such that $\hbar^{2} \omega_{p e}^{2} / m_{e}^{2} c^{2} v_{t}^{2}$ approaches unity. However, increasing the density to fulfill this, the minimum velocity spread will also increase, and when this parameter approaches unity we have also reached the regime of a relativistic Fermi velocity. Nevertheless, one can see that the spin has a certain significance even if the dimensionless parameter of this example is much smaller than unity. Firstly, the spin term makes the dispersion relation slightly anisotropic, since the group velocity is not parallel to the wave vector anymore. Secondly, in the given approximation the group dispersion comes solely from the spin effect. Finally, provided the omitted cyclotron resonances lies far out in the thermal tail, the imaginary contribution is determined by the spin term, and is given by

$$
\gamma \equiv \operatorname{Im}(\omega)=\left(\frac{k^{2} c^{2}}{1+\omega_{p i}^{2} / \omega_{c i}^{2}}\right)^{1 / 2} \frac{\pi \sin ^{2} \alpha \hbar^{2} \omega_{p e}^{2}}{2 m_{e}^{2} c^{2} v_{t e}^{2}} \exp \left(-\frac{(\operatorname{Re} \omega)^{2}}{k_{z}^{2} v_{t}^{2}}\right)
$$

when the influence of the spin term is small. Thus we can conclude that there are several possibilities for spin to be of significance, even if all dimensionless parameters determining the magnitude of the spin contribution are much smaller than unity.

As a final comment, let us make a few comments regarding the terms in Eq. (12) with denominators $\omega-k_{z} v_{s} \pm$ $\left(\omega_{c e} \pm \omega_{c g}\right)$. As pointed out in Ref. [10], these terms may give raise to wave modes with frequencies $\omega \approx\left|\omega_{c g}-\omega_{c e}\right|$, which is a completely new effect due to spin, that can survive even when all dimensionless parameters $d_{1}-d_{6}$ are small. In the next section we will demonstrate from the general result, Eq. (12), that the presence of the denominators $\omega-k_{z} v_{s} \pm\left(\omega_{c e} \pm \omega_{c g}\right)$ has further consequences for the transition from wave damping to instability. 


\section{SPIN INSTABILITY - AN EXAMPLE}

In order to illustrate the usefulness of (12), we will evaluate the imaginary contribution associated with the denominators $\omega-k_{z} v_{z} \pm\left(\omega_{c g}-\omega_{c}\right)$. To compute such terms explicitly, we must pick a specific form of the unperturbed distribution function. As we here have not been interested in classical instabilities, that can be induced by a velocityspace non-equilibrium, we have assumed the distribution function to be isotropic, i.e. $f_{0}=f_{0}\left(v^{2}\right)$. However, as we will see below, this does not remove all possibilities for free energy to be present in the background distribution function. Specifically, in case the number of particles in the two spin states does not correspond to thermodynamic equilibrium, we may find that certain wave modes may become unstable. To illustrate this idea we let the unperturbed distribution function $F_{0 \pm}$ be of the form

$$
F_{0 \pm}=n_{0}\left(\frac{m_{e}}{2 \pi k_{B} T_{\mathrm{kin}}}\right)^{3 / 2} \exp \left[-\frac{m_{e} v^{2}}{2 k_{B} T_{\mathrm{kin}}}\right] G_{ \pm}\left(T_{\mathrm{sp}}\right)
$$

where $G_{ \pm}\left(T_{\mathrm{sp}}\right)$ is proportional to the number of particles in spin-up and spin-down states respectively and can be written as

$$
G_{\nu}\left(T_{\mathrm{sp}}\right) \equiv \frac{\exp \left(\nu \mu_{e} B_{0} / k_{B} T_{\mathrm{sp}}\right)}{\exp \left(\mu_{e} B_{0} / k_{B} T_{\mathrm{sp}}\right)+\exp \left(-\mu_{e} B_{0} / k_{B} T_{\mathrm{sp}}\right)}
$$

where we have introduced the parameter $T_{\mathrm{sp}}$ that can be interpreted as a spin temperature, in addition to the kinetic temperature $T_{\text {kin }}$ of the ordinary Maxwellian velocity dependence. If $T_{\mathrm{sp}}=T_{\text {kin }}=T$ we get a thermodynamic equilibrium distribution with the common temperature $T$, and if we let $T_{\mathrm{sp}} \rightarrow \infty$, we get the same number of particles in the two spin states. We note that for most plasmas, the energy difference between the high-energy and low-energy spin state is small ( i.e. typically $\mu_{e} B_{0} / k_{B} T \ll 1$ ), but nevertheless even a small energy energy difference can be crucial, as we will see below.

Next we assume that the plasma parameters $\left(B_{0}, n_{0}, T_{\text {kin }}\right)$ correspond to a classical regime, such that the real part of the frequency $\omega_{r}\left(k_{\perp}, k_{z}\right)$ is given by the classical terms in (12) to a good approximation. Furthermore, we assume that all resonant electron velocities $v_{z}= \pm\left(\omega-n \omega_{c}\right) / k_{z}$ corresponding to the classical terms lies very far out in the thermal tail. The dominant imaginary contribution to the dispersion relation may then come from the spin terms $X_{i j}^{(s p)}$ and $Z_{i j}^{(s p)}$ with resonant particle velocities $v_{z}=\left(\omega \pm\left|\Delta \omega_{c}\right|\right) / k_{z}$ (corresponding to $a= \pm 1$ in the conductivity tensor (12) ) with $\left|\Delta \omega_{c}\right|=\left|\omega_{c g}-\omega_{c e}\right|$. Furthermore, letting the Larmor radius be smaller than $k_{\perp}^{-1}$ (such that the Bessel functions can be Taylor expanded), we can compute the pole contributions from $X_{i j}^{(s p)}$ and $Z_{i j}^{(s p)}$ with the help of well known properties of the plasma dispersion function; $\operatorname{Im} Z(\zeta)=i \pi^{1 / 2} \exp \left(-\zeta^{2}\right)$. Since the lower value of the resonant velocity occurs for $v_{z}=\left(\omega-\left|\Delta \omega_{c}\right|\right) / k_{z}$ we concentrate on the contribution from $X_{i j}^{(s p)}$ (the contribution from $Z_{i j}^{(s p)}$ at $v_{z}=\left(\omega+\left|\Delta \omega_{c}\right|\right) / k_{z}$ can be computed analogously). The result is then

$$
\begin{aligned}
\operatorname{Im}\left(\sigma_{i j}\right) & =n_{0} \sqrt{\frac{2 \pi k_{B} T_{\mathrm{kin}}}{m_{e}}} \frac{\mu_{e}^{2} k_{\perp}^{2}}{4 \hbar k_{z} \omega_{c}^{2} \omega}\left(-\tanh \left(\frac{\mu_{e} B_{0}}{k_{B} T_{\mathrm{sp}}}\right)+\frac{\hbar \omega_{c e}}{2 k_{B} T_{\mathrm{kin}}}\right) \exp \left[\frac{-m_{e}\left(\omega-\left|\Delta \omega_{c}\right|\right)^{2}}{2 k_{z}^{2} k_{B} T_{\mathrm{kin}}}\right] M_{i j} \\
& =A_{i j}\left(T_{\mathrm{kin}}, n_{0}, B_{0}\right)\left(\tanh \left(\frac{g}{2} \tilde{a} \tilde{T}\right)-\tilde{a}\right)
\end{aligned}
$$

where we have noted that $\sum_{\nu} G_{\nu}=1$ and $\sum_{\nu} \nu G_{\nu}=\tanh \left(\mu_{e} B_{0} / k_{B} T_{\mathrm{sp}}\right)$, and we have defined $\tilde{a}=\frac{\hbar\left|\omega_{c e}\right|}{2 k_{B} T_{\mathrm{kin}}}$ and $\tilde{T}=T_{\text {kin }} / T_{\mathrm{sp}}$. In Fig. 1 we plot $\operatorname{Im}\left(\sigma_{i j}\right)$ as a function of $\tilde{T}$ for some different values of $\tilde{a}$. For the case of thermodynamic equilibrium, $T_{\mathrm{sp}}=T_{\mathrm{kin}}$, the fact that $g / 2>1$ ensures that $\operatorname{Im}\left(\sigma_{i j}\right)>0$ (at least for not too strong magnetic fields, i.e. small values of $\tilde{a}$ ). In this case the contribution to the conductivity tensor from the poles gives raise to a small damping rate. This applies independently of the polarization of the mode under consideration. However, we need only perturb the spin temperature a little from equilibrium $\left(T_{\mathrm{sp}}>T_{\mathrm{kin}}\right)$ for the system to become unstable. The weak damping then turns to a weak growth rate of all modes where the resonant velocities $v_{z}=\left(\omega-\Delta \omega_{c}\right) / k_{z}$ does not fall too far out in the tail of the distribution. We stress that this may apply to all sorts of ion-cyclotron modes, as $\Delta \omega_{c}$ is of the same order as the ion-cyclotron frequency. It is not hard to imagine situations where the plasma state deviates from thermodynamic equilibrium, in which case the sign of the pole contribution may change. For example, if particles are flowing from a region of weak magnetic field to a region of strong magnetic field, the inflowing particles will be more evenly distributed between spin-up and spin-down states than that of the local thermodynamic state. Hence the spin-distribution will be more evenly distributed than for $T_{\mathrm{sp}}=T_{\mathrm{kin}}$, i.e. we will get $T_{\mathrm{sp}}>T_{\mathrm{kin}}$. The same situation will also occur if the external magnetic field is increased slowly, but at a rate slightly faster than the spin-states can relax to thermodynamic equilibrium. 


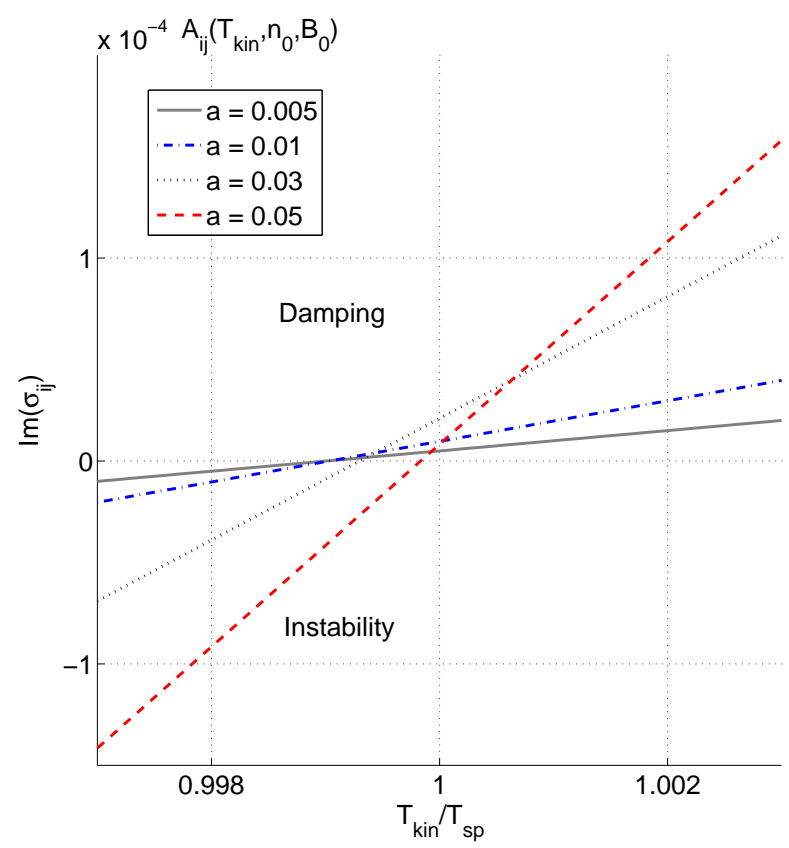

FIG. 1: (Color online) The imaginary contribution of $X_{i j}^{(s p)}$ to $\sigma_{i j}$ plotted as a function of $T_{\mathrm{sp}} / T_{\mathrm{kin}}$ for different values of $\tilde{a}$. A positive value corresponds to damping while a negative value gives rise to a instability.

It may be noted that if $\tilde{a}$ is sufficiently large (corresponding to a strongly magnetized systems), the result (17) predicts that the system will be unstable already at thermal equilibrium. However, as $\tilde{a}$ increases, the effect of Landau quantization will become important (which affects the perpendicular kinetic energy distribution, see e.g. [6]), and hence the background distribution function will no longer be isotropic. The validity of Eq. (17) is therefore restricted to small values of $\tilde{a}$. Finally, let us make an estimate for the growth rate for the case of ion-cyclotron Alfvén waves with $\omega \sim \omega_{c i} \sim k_{z} c_{A} \sim k_{\perp} c_{A}$. We let the the temperature ratio be well above the threshold for instability (i.e. $T_{\mathrm{sp}} / T_{\text {kin }} \sim 2$ ), and let $\tilde{a} \ll 1$ (such that the isotropic distribution function is justified). The normalized growth rate $\gamma / \omega$ found from 17 is then of the order

$$
\frac{\gamma}{\omega} \sim \frac{\hbar^{2} \omega_{p e}^{2}}{m_{e} m_{i} c_{A}^{2}}
$$

if the let the resonance approach the bulk of the distribution in order to find the maximum groth rate.

\section{CONCLUSION}

In the present paper we have studied the regime of long spatial scales in an otherwise fully quantum mechanical kinetic model. The assumption of long spatial scales (compared to the characteristic de Broglie wavelength) makes the model reminiscent of semi-classical theory. In particular, the distribution function behaves as if particles are effectively localized spatially, and unlike the Wigner function the distribution function is always positive in our case. Still, the impossibility for the spin vector of individual particles to have a localized probability distribution in spin space keeps certain quantum features in the evolution equation (11). The linearized theory is solved in a magnetized plasma for a homogeneous background, which leads to the general conductivity tensor, Eq. (12), that includes both the contribution from the free current density as well as that from the magnetization current due to the spin. This is the main result of the paper. The main restriction in applying Eq. (12) is that the velocity distribution is assumed to be isotropic. Specifically we have used $f_{0}=f_{0}\left(v^{2}, \theta_{s}\right)=(1 / 4 \pi)\left[F_{0+}\left(v^{2}\right)\left(1+\cos \theta_{s}\right)+F_{0-}\left(v^{2}\right)\left(1-\cos \theta_{s}\right)\right]$. Note that, by contrast, the spin distribution of the unperturbed distribution function is the most general time-independent solution for a constant magnetic field background.

In section III we have discussed the quantum-classical boundary, which is considerably more complicated when spin effects are included. In particular, as deduced from the dimensionless parameter $d_{3}-d_{6}$, spin effects may in certain cases remain also in a plasma of modest temperature and density. Furthermore, effects due to the new types 
of wave-particle resonances may be of signficance independent of the dimensioneless parameters. In section IV, we have found that such resonances is one of the key ingredients in a new type of instability, somewhat reminiscent of the Weibel-instability. As is wellknown (see e.g. [20]), in the case of Weibel-instabilites the instabilities arise from a difference in perpendicular and parallell kinetic temperatures. Here, the deviation in the spin temperature from a common (isotropic) kinetic temperature is the source of the instability. Furthermore we note that a very small deviation of $T_{\mathrm{kin}} / T_{\mathrm{sp}}$ from unity is sufficient to drive an instability.

Although the present result (12) is relatively general, there is still several interesting extensions that could be made;

1. Generalization to an arbitrary (non-isotropic) background velocity distribution.

2. Inclusion of the spin-orbit coupling.

3. Using the full evolution of Ref. [6], including the short-scale physics (i.e. shorter than the characteristic de Broglie wavelength).

4. Inclusion of collisional effects.

Furthermore, a thorough evaluation of (12), that must be done numerically, could possibly reveal new and interesting possibilities, involving e.g. new wave modes.

\section{Appendix A: Some useful relations}

The integrals (A1a)-(A1c) are very useful when performing the $\varphi_{s}$ and $\varphi_{v}$ integrations in Eqs. (7b), (10) and (11),

$$
\begin{aligned}
& \int_{0}^{2 \pi} d \varphi \exp (i n \varphi) \cos (\varphi)=\left\{\begin{array}{l}
\pi \text { for } n= \pm 1 \\
0 \text { for } n \neq \pm 1
\end{array}\right. \\
& \int_{0}^{2 \pi} d \varphi \exp (i n \varphi) \sin (\varphi)=\left\{\begin{array}{ccc} 
\pm i \pi & \text { for } & n= \pm 1 \\
0 & \text { for } & n \neq \pm 1
\end{array}\right. \\
& \int_{0}^{2 \pi} d \varphi \exp (i n \varphi)=\left\{\begin{array}{ccc}
2 \pi & \text { for } & n=0 \\
0 & \text { for } & n \neq 0 .
\end{array}\right.
\end{aligned}
$$

When performing the $\theta_{s}$ integration in going from Eqs. (10) and (11) to (12), it is useful to note that integrals on the form

$$
\int_{0}^{\pi} d \theta(\cos \theta)^{n}(\sin \theta)^{m}
$$

vanish for all odd integer values of $n$ independent of the integer value of $m$.

\section{Appendix B: The Hermitian structure of the conductivity tensor}

The Hermitian structure of the conductivity tensor $\left(\sigma_{i j}=-\sigma_{i j}^{*}\right)$ is not transparent from Eqs. (10) and (11). The conductivity tensor will for instance not be recognized to have a Hermitian structure until after the $\theta_{s}$-integration is performed. Below we list some further identities that have been used in obtaining $\sigma_{i j}$ on the form written in Eq. (12).

To match the terms $\sigma_{x y}$ with $\sigma_{y x}$, it is found that an integral of the form

$$
\sum_{a=-\infty}^{\infty} \int_{0}^{\infty} \frac{a \mathcal{J}_{a}^{2}}{\omega-k_{z} v_{z}-a \omega_{c e}} \frac{\partial f_{0}}{\partial v^{2}} v_{\perp} d v_{\perp}
$$

must match the integral

$$
\begin{aligned}
& \sum_{a=-\infty}^{\infty} \int_{0}^{\infty} \frac{a}{\omega} \frac{\left(a \omega_{c e}+k_{z} v_{z}\right) \mathcal{J}_{a}^{2}}{\omega-k_{z} v_{z}-a \omega_{c e}} \frac{\partial f_{0}}{\partial v^{2}} v_{\perp} d v_{\perp} \\
= & \sum_{a=-\infty}^{\infty} \int_{0}^{\infty}\left(-a \mathcal{J}_{a}^{2}+\frac{a \mathcal{J}_{a}^{2}}{\omega-k_{z} v_{z}-a \omega_{c e}}\right) \frac{\partial f_{0}}{\partial v^{2}} v_{\perp} d v_{\perp} .
\end{aligned}
$$


For the terms to match, the following integral must vanish;

$$
\begin{aligned}
\sum_{a=-\infty}^{\infty} \int_{0}^{\infty} a \mathcal{J}_{a}^{2} \frac{\partial f_{0}}{\partial v^{2}} v_{\perp} d v_{\perp} & =\frac{1}{2} \sum_{a=-\infty}^{\infty} \int_{0}^{\infty} a \mathcal{J}_{a}^{2} \frac{\partial f_{0}}{\partial v_{\perp}} d v_{\perp} \\
& =-\frac{1}{2} \int_{0}^{\infty} \sum_{a=-\infty}^{\infty} a \mathcal{J}_{a}\left(\mathcal{J}_{a-1}-\mathcal{J}_{a+1}\right) f_{0} d v_{\perp} \\
& =-\frac{1}{2} \int_{0}^{\infty} \sum_{n=1}^{\infty}\left[n \mathcal{J}_{n}\left(\mathcal{J}_{n-1}-\mathcal{J}_{n+1}\right)-n \mathcal{J}_{-n}\left(\mathcal{J}_{-(n+1)}-\mathcal{J}_{-(n-1)}\right)\right] f_{0} d v_{\perp} \\
& =0
\end{aligned}
$$

where we have integrated by parts, summed over all integers $a$ and used the relation $\mathcal{J}_{-n}=(-1)^{n} \mathcal{J}_{n}$. Thus, since the integral (B33) indeed vanish, we have $\sigma_{x y}=-\sigma_{y x}^{*}$ as expected.

In a similar manner, we find that the following integral must vanish for $\sigma_{y z}$ to match $\sigma_{z y}$;

$$
\int_{-\infty}^{\infty} v_{z} \frac{\partial f_{0}}{\partial v^{2}} d v_{z}=0
$$

This is trivially satisfied under the assumption $f_{0}\left(v^{2}\right)$ since this is a product of an odd and an even function integrated from $-\infty$ to $\infty$.

\section{Appendix C: The short Larmor radius limit}

Below we consider the short Larmor radius limit of the conductivity tensor (12), i.e. the case where the Bessel argument is small $k_{\perp} v_{\perp} /\left|\omega_{c e}\right| \ll 1$. Since we have assumed an isotropic distribution for the plasma $\left(f_{0}\left(v^{2}\right)\right)$, we note that

$$
\frac{\partial F_{0}}{\partial v^{2}}=\frac{1}{2 v_{z}} \frac{\partial F_{0}}{\partial v_{z}} .
$$

With this noted, we can integrate by parts with respect to $v_{z}$ to eliminate the derivatives of $F_{0}$ in the conductivity tensor (12). We encounter integrals on the form

$$
\begin{aligned}
\int_{-\infty}^{\infty} \frac{v_{z}^{n}}{\Omega-k_{z} v_{z}} \frac{\partial F_{0}}{\partial v^{2}} d v_{z} & =\frac{1}{2} \int_{-\infty}^{\infty} \frac{v_{z}^{n-1}}{\Omega-k_{z} v_{z}} \frac{\partial F_{0}}{\partial v_{z}} d v_{z} \\
& =-\frac{1}{2} \int_{-\infty}^{\infty} F_{0}\left(\frac{(n-1) v_{z}^{n-2}}{\Omega-k_{z} v_{z}}+\frac{k_{z} v_{z}^{n-1}}{\left(\Omega-k_{z} v_{z}\right)^{2}}\right) d v_{z}
\end{aligned}
$$

where $n$ may take the integer values $n=0,1,2$. Here $\Omega \equiv \omega-a \omega_{c e}-b \omega_{c g}$ with the integer values of $a$ and $b$ not being specified. Next, we expand the Bessel functions in $v_{\perp} k_{\perp} / \omega_{c e}$ and perform the summation over all integer values of $a$. Terms proportional to $k_{\perp} v_{\perp} / \omega_{c e}$ are considered small and are dropped. The conductivity tensor then becomes:

$$
\sigma_{i j}=\sum_{\nu=+,-}\left[x_{(\nu) i j}^{(\mathrm{sp})}+y_{(\nu) i j}^{(\mathrm{cl})}+y_{(\nu) i j}^{(\mathrm{sp})}+z_{(\nu) i j}^{(\mathrm{sp})}\right]
$$

where

$$
y_{(\nu) i j}^{(\mathrm{cl})}=\frac{q_{e}^{2}}{m_{e}} \int F_{0 \nu}\left[\frac{\omega}{\left(\omega-k_{z} v_{z}\right)^{2}} \times\left(\begin{array}{ccc}
0 & 0 & 0 \\
0 & 0 & 0 \\
0 & 0 & i
\end{array}\right)+\frac{1}{4} \sum_{a= \pm 1} \frac{\omega-2 k_{z} v_{z}-a \omega_{c e}}{\left(\omega-k_{z} v_{z}-a \omega_{c e}\right)^{2}} \frac{v_{1}^{2}}{v_{z}^{2}} \times\left(\begin{array}{ccc}
-i & a & 0 \\
-a & -i & 0 \\
0 & 0 & 0
\end{array}\right)\right] d^{3} v
$$

is the classical contribution, and the spin contributions are

$$
y_{(\nu) i j}^{(\mathrm{sp})}=\mu_{e} \frac{q_{e}}{m_{e}} \int F_{0 \nu}\left[\frac{1}{\left(\omega-k_{z} v_{z}\right)^{2}} \times\left(\begin{array}{ccc}
0 & 0 & 0 \\
0\left(i \frac{\mu_{e}}{q_{e}} \frac{k_{\perp}^{2} k_{z}^{2}}{\omega} \nu i \frac{1}{2} \frac{v_{\perp}^{2}}{v_{z}^{2}} \frac{k_{\perp}^{2}\left(\omega-2 k_{z} v_{z}\right)}{\omega_{c e}}\right) & \nu k_{\perp} k_{z} \\
0 & -\nu k_{\perp} k_{z} & 0
\end{array}\right)\right.
$$




$$
\left.-\frac{1}{4} \frac{k_{\perp}^{2}}{\omega_{c e}} \sum_{a= \pm 1} \frac{\omega-2 k_{z} v_{z}-a \omega_{c e}}{\left(\omega-k_{z} v_{z}-a \omega_{c e}\right)^{2}} \frac{v_{\perp}^{2}}{v_{z}^{2}} \times\left(\begin{array}{ccc}
0 & -\nu a & 0 \\
\nu a & \left(i a \frac{\mu_{e}}{q_{e}} \frac{k_{\perp}^{2}}{\omega}+\nu i \frac{\left(\omega+a \omega_{c e}\right)}{\omega}\right) & 0 \\
0 & 0 & 0
\end{array}\right)\right] d^{3} v
$$

together with

$$
\begin{aligned}
x_{(\nu) i j}^{(\mathrm{sp})}+z_{(\nu) i j}^{(\mathrm{sp})} & =\sum_{b= \pm 1}\left\{i \frac { \mu _ { e } ^ { 2 } } { \hbar \omega } \int F _ { 0 \nu } \left[\frac{-\nu b}{\omega-k_{z} v_{z}-b \omega_{c g}}-\frac{1}{2} \frac{\hbar}{m} \frac{k_{z}^{2}}{\left(\omega-k_{z} v_{z}-b \omega_{c g}\right)^{2}}\right.\right. \\
& \left.\left.+\sum_{a= \pm 1} a \frac{1}{8} \frac{\hbar}{m_{e}} \frac{k_{\perp}^{2}}{\omega_{c e}} \frac{v_{\perp}^{2}}{v_{z}^{2}} \frac{\omega-2 k_{z} v_{z}-a \omega_{c e}-b \omega_{c g}}{\left(\omega-k_{z} v_{z}-a \omega_{c e}-b \omega_{c g}\right)^{2}}\right] M_{i j}^{(b)} d^{3} v\right\}
\end{aligned}
$$

where $M_{i j}^{(-1)} \equiv M_{i j}$ with $M_{i j}$ being defined as before, and $M_{i j}^{(1)} \equiv M_{i j}^{*}$.

We may note that we only encounter four kinds of unique integrals in the conductivity tensor (C2);

$$
\begin{aligned}
& \int F_{0} \frac{1}{\Omega-k_{z} v_{z}} d^{3} v \\
& \int F_{0} \frac{v_{\perp}^{2}}{v_{z}^{2}} \frac{1}{\left(\Omega-k_{z} v_{z}\right)^{2}} d^{3} v \\
& \int F_{0} \frac{v_{\perp}^{2}}{v_{z}} \frac{1}{\left(\Omega-k_{z} v_{z}\right)^{2}} d^{3} v \\
& \int F_{0} \frac{1}{\left(\Omega-k_{z} v_{z}\right)^{2}} d^{3} v .
\end{aligned}
$$

For the special case when the cyclotron resonances are far out in the thermal tail, the conductivity tensor (C2) may be significantly simplified. This is, however, trivial to obtain from (C2) and will therefore not be pursued further here.

[1] G. Manfredi, Fields Inst. Comm. 46, 263 (2005).

[2] P. K. Shukla and B. Eliasson, Phys.-Usp. 53, 51 (2010).

[3] F. Haas, G. Manfredi, and M. R. Feix, Phys. Rev. E 62, 2763 (2000).

[4] L. G. Garcia, F. Haas, L. P. L. de Oliviera, and J. Goedert, Phys. Plasmas 12, 012302 (2005).

[5] P. K. Shukla and B. Eliasson, Phys. Rev. Lett. 96, 245001 (2006).

[6] J. Zamanian, M. Marklund, and G. Brodin, New J. Phys. 12, 043019 (2010).

[7] M. Marklund and G. Brodin, Phys. Rev. Lett. 98, 025001 (2007).

[8] G. Brodin, M. Marklund, and G. Manfredi, Phys. Rev. Lett. 100, 175001 (2008).

[9] M. Marklund, G Brodin, L. Stenflo and C. S. Liu, Europhys. Lett. 84, 17006 (2008).

[10] G. Brodin, M. Marklund, J. Zamanian, A. Ericsson, and P. L. Mana, Phys. Rev. Lett. 101, 245002 (2008).

[11] G. Brodin, M. Marklund and J. Zamanian p. 280-290 in New developments in nonlinear plasma physics. Eds. B. Eliasson and P. K. Shukla, AIP conf. proce. No 1188, (AIP, New York, 2009).

[12] G. Manfredi and P.-A. Hervieux, Appl. Phys. Lett. 91, 061108 (2007).

[13] H. A. Atwater, Sci. Am. 296, 56 (2007).

[14] S. A. Wolf, D. Awschalom, R. A. Buhrman, et al., Science 294, 1488 (2001).

[15] C. Kouveliotou, S. Dieters and T. Strohmayer et al., Nature 393, 235 (1998); D. M. Palmer, S. Barthelmy, and N. Gehrels, Nature 434, 1107 (2005); A. K. Harding and D. Lai, Rep. Prog. Phys. 69, 2631 (2006).

[16] M. P. Robinson, B. Laburthe Tolra, M. W. Noel, T. F. Gallagher, and P. Pillet, Phys. Rev. Lett. 85, 4466 (2000).

[17] S. H. Glenzer et al., Phys. Rev. Lett. 98, 065002 (2007).

[18] S. C. Cowley, R. M. Kulsrud, and E. Valeo, Phys. Fluids 29, 430 (1986).

[19] R. M. Kulsrud, E. J. Valeo, and S. C. Cowley, Nucl. Fusion 26, 1443 (1986).

[20] D. G. Swanson, Plasma Waves (Taylor \& Francis, 2003).

[21] It should be noted that we have defined the conductivity tensor to include all contributions to the current density, i.e. the conductivity tensor includes the free current density (due to the Lorentz force and the magnetic dipole force) as well as the contributions from the magnetization current density.

[22] When performing this estimate, we have ignored the spin-contribution from the first term in $X_{i j}^{(\mathrm{sp})}\left(\right.$ and $\left.Z_{i j}^{(\mathrm{sp})}\right)$. This is valid e.g. if there is equal number of spin-up and spin-down populations, in which case this contribution vanishes. 